\title{
La educación no escolarizada como nueva opción para el bachillerato
}

\author{
Non-school education as a new option for high school \\ Kleber Antonio Luna Altamirano. ${ }^{1}$, Natalia Aguilar Aguilar. ${ }^{2}$, Jessenia Valeria Moreno \\ Guanaquiza. ${ }^{3} \&$ Diego Alexander Santos Medina. ${ }^{4}$
}

\begin{abstract}
Non-school education is an educational option that today is becoming stronger in the urban area of the city of Cuenca-Ecuador, it is usually aimed at those parents who choose to educate their children outside a public secondary education institution or private, known as family education, and for people who have not had the opportunity to complete their studies. The problem of the investigation is the lack of knowledge on the part of the society of this modality of study, and that is stipulated in the Organic Law of Intercultural Education (LOEI) of Ecuador. The objective of this study is to design an information and communication plan aimed at the inhabitants of the Octavio Cordero Palacios Parish, belonging to the city of CuencaEcuador, on this new modality of study and thereby encourage the authorities of secondary education for its continuity in other sectors. In the methodological, descriptive research with a quantitative approach, supporting their information through the survey technique. The results explain that most of the population in which the surveys were applied do not know about nonschool education and if they are willing to put into practice the fact of educating their children at home, they also know that this type of education is legal.
\end{abstract}

Keywords: Ignorance, unschooled education, legal framework.

\footnotetext{
${ }^{1}$ Doctor (c) en Ciencias Sociales Mención Gerencia Universidad del Zulia República Bolivariana de Venezuela, Profesor Investigador Unidad Académica de Administración Universidad Católica de Cuenca, klunaa@ucacue.edu.ec

2 Estudiante de la carrera de Contabilidad y Auditoría en la Unidad Académica de Administración de la Universidad católica de Cuenca, naty@est.ucacue.edu.ec

3 Estudiante de la carrera de Contabilidad y Auditoría en la Unidad Académica de Administración de la Universidad Católica de Cuenca, jessenia@est.ucacue.edu.ec

4 Estudiante de la carrera de Contabilidad y Auditoría en la Unidad Académica de Administración de la Universidad Católica de Cuenca, diego@est.ucacue.edu.ec
} 


\section{Resumen}

La educación no escolarizada es una opción educativa que hoy en día está tomando mayor fuerza en la zona urbana de la ciudad de Cuenca-Ecuador, por lo general va dirigida para aquellos padres que elijan educar a sus hijos fuera de una institución de educación media pública o privada, conocida como educación familiar, y para personas que no han tenido la oportunidad de culminar sus estudios. El problema de la investigación es el desconocimiento por parte de la sociedad de esta modalidad de estudio, y que se encuentra estipulada en la Ley Orgánica de Educación Intercultural (LOEI) del Ecuador. El objetivo de este estudio, es diseñar un plan de información y comunicación dirigido a los habitantes de la Parroquia Octavio Cordero Palacios, perteneciente a la ciudad de Cuenca-Ecuador, sobre esta nueva modalidad de estudio y con ello incentivar a las autoridades de educación media para su continuidad en otros sectores. En lo metodológico, la investigación de tipo descriptivo con un enfoque cuantitativo, soportando su información a través de la técnica de la encuesta. Los resultados explican que la mayoría de la población en la que se aplicó las encuestas no conocen sobre la educación no escolarizada y si están dispuestos a poner en práctica el hecho de educar a sus hijos en casa, además conocen que este tipo de educación está bajo un marco jarico legal.

Palabras Clave: Desconocimiento, educación no escolarizada, marco legal.

\section{Introducción}

La educación es un derecho que todos los seres humanos deben tener desde su uso de razón, esta es aprobada por la LOEI. La presente investigación trata a cerca de una alternativa educativa denominada educación no escolarizada. La educación no escolarizada es un proceso continuo y metódico mediante el cual las personas interesadas adquieren o mejoran sus conocimientos y habilidades mediante cursos de corta duración, esta se puede llegar a convertir en una herramienta formativa de suma importancia (M.E.E, 2019). El problema abordado en esta investigación se centra en el desconocimiento por parte de la sociedad sobre esta nueva alternativa de estudio. Según Moore y Moore "las experiencias más profundas y educativas son el contacto no verbal con las cosas" (Moore y Moore, 1995, p.75). El objetivo de la investigación es diseñar un plan de información y comunicación el cual consiste en establecer de forma clara como y cuando se va a dar a conocer a la sociedad de esta nueva modalidad de estudio. Molero (2005) afirma "el Plan de Comunicación es el esqueleto, la base que permite practicar una comunicación institucional profesional (...)” (p.4). 
En lo metodológico, la investigación es de tipo descriptivo, se utiliza para describir las características de una población o fenómeno en estudio. No responde preguntas sobre cómo, cuándo y por qué ocurrieron las características, más bien aborda la cuestión del qué. Meyer y Deobold (2006) sostienen "la investigación descriptiva consiste en llegar a conocer las situaciones, costumbres y actitudes predominantes a través de la descripción exacta de las actividades, objetos, procesos y personas" (p.1). El enfoque del estudio es cuantitativo, el cual recoge y analiza datos cuantitativos sobre variables y estudia las propiedades y fenómenos de dicho enfoque. Sampieri (2014) afirma "El enfoque cuantitativo (que representa, como dijimos, un conjunto de procesos) es secuencial y probatorio. Cada etapa precede a la siguiente y no podemos "brincar" o eludir pasos. El orden es riguroso, aunque desde luego, podemos redefinir alguna fase" (p.37).

El método a aplicar será el deductivo, ya que es una estrategia de razonamiento empleada para deducir conclusiones lógicas a partir de una serie de premisas o principios. Maya (2014) afirma "el método deductivo es una forma de razonamiento que parte de una verdad universal para obtener conclusiones particulares" (p. 14).

Como técnica para dar fuerza a la investigación, se utilizará la encuesta, esta es una de las estrategias más utilizadas en el área de la investigación, dado que favorece a la obtención de datos fundamentales para el análisis de diversas temáticas, permitiendo una mayor eficacia y rapidez en el procedimiento.

Las encuestas son instrumentos de investigación descriptiva que precisan identificar a priori las preguntas a realizar, las personas seleccionadas en una muestra representativa de la población, especificar las respuestas y determinar el método empleado para recoger la información que se vaya obteniendo (Trespalacios, Vázquez y Bello 2005, p. 96)

En la presente investigación se desarrolla preguntas para obtener información relevante para el diseño del plan de comunicación.

El estudio, está constituida como primera parte en el estado del arte, mismo que da soporte teórico con la presencia de autores que den mayor relevancia a la investigación. En la parte de la metodología se plantea la construcción de un plan de comunicación que ayudara a informar a la sociedad sobre la educación no escolarizada. Como siguiente punto se platea los resultados los cuales apoyaran a la sociedad a informarse sobre la educación no escolarizada y como conclusión se presentará ante la sociedad los beneficios de este nuevo tipo de educación y con ello se logrará dar solución al problema encontrado. 


\section{Estado del arte}

Al diseñar un plan de comunicación para la difusión de la información en la parroquia Octavio Cordero Palacios de la ciudad de Cuenca-Ecuador, es de suma importancia la contribución de autores relevantes, entre ellos y puede citar a Valle (2012) quien explica que este tipo de educación, aunque tiene un bajo número de personas afectas, está teniendo un gran auge, aunque la sociedad no esté al tanto de este nuevo tipo de educación. El mismo autor, Valle (2012) propone que de igual manera buscar un entorno en el cual pueda desarrollar los principios democráticos en respecto a lo personal, las familias al acceder a este tipo de educación asumen de manera integral la educación de los niños y niñas. Por su parte, Rodríguez (2019) opina la familia se convierte en la institución que transmite valores y principios, que se ocupa del desarrollo afectivo y emocional de los pequeños, y que además asume otras funciones en la transmisión de las habilidades y los conocimientos necesarios para la formación y para el completo desarrollo de los menores.

La implementación de nuevas ideas o métodos de educación han contribuido a la mejora de la educación, Farrel, Manion, y Rincón (2017) opina que algunas maneras de educar fueron concebidas originalmente en la Europa Oriental a inicios del siglo XIX y algunos de esos aspectos se han convertido en formas universales de estudio, con los adelantos en la educación se implanta hoy en día diálogos.

Sierra (2010) expone que gracias a la educación se implementa hoy en día espacios abiertos a la producción de conocimientos, al debate, por todos estos puntos se ha creado instituciones sociales. Tanaro (2006) sostiene que las instituciones sociales se crean para que ellas puedan responder a las necesidades de un grupo social y por lo tanto se han caracterizado en estar en momentos históricos. Guitierrez (2014) expresa que la inclusión en las épocas de antes era algo incierto puesto que las no ponían en práctica la poca información que obtuvieron. Bravo (2009) indica que se presentan lagunas en la educación formal y existen preocupaciones como las de ahogar el espíritu creativo de los pequeños presionándoles a realizar actividades definidas para su edad.

El plan de comunicación tiene muchas facetas, Fonseca (2005) afirma "Los modelos de comunicación son útiles para representar la relación y dinámica entre los elementos del proceso comunicativo. Algunos exponen más que otros. No obstante, en todos ellos se enfatizan tres componentes básicos para que se establezca una comunicación: emisor, mensaje y receptor" (p.155-156). 
La Ley Orgánica de Educación Intercultural del Ecuador (LOEI), señala en su Art. 38.Educación escolarizada y no escolarizada. - El Sistema Nacional de Educación ofrece dos tipos de educación escolarizada y no escolarizada con pertinencia cultural y lingüística." La educación no escolarizada brinda la oportunidad de formación y desarrollo de los ciudadanos a lo largo de la vida y no está relacionada con los currículos determinados para los niveles educativos (...)".

Los autores descritos, aportan a un mejor desarrollo del plan de comunicación, ya que estos proporcionan varias alternativas para brindar información a la sociedad, y con ello llevar a cabo una estrategia para informar a la ciudadanía de forma correcta lo que es la educación no escolarizada y motivar a que lo pongan en práctica en la Parroquia Octavio Cordero Palacios.

\section{METODOLOGIA}

La presente investigación es de tipo descriptivo, con un enfoque cuantitativo por lo que se aplica el método deductivo para diseñar el plan de comunicación, la información obtenida por medio de una encuesta dirigida a 30 personas de la parroquia Octavio Cordero Palacios, los cuales están interesados en obtener un título de nivel medio, con el propósito de dar mayor veracidad a la información, a continuación, se detalla a quienes está dirigida la encuesta.

\section{RESULTADOS}

La población de la Parroquia Octavio Cordero Palacios, está representado por 30 personas a los cuales se aplicó la encuesta, sus edades fluctúan entre 25-40 años, quienes no poseen un título de tercer nivel, pero si están interesados en obtenerlo bajo esta modalidad, y a más de ello están dispuestos a educar a sus hijos en casa. La información más importante de las encuestas se detallará a continuación:

Figura 1. Información sobre la Educación no escolarizada

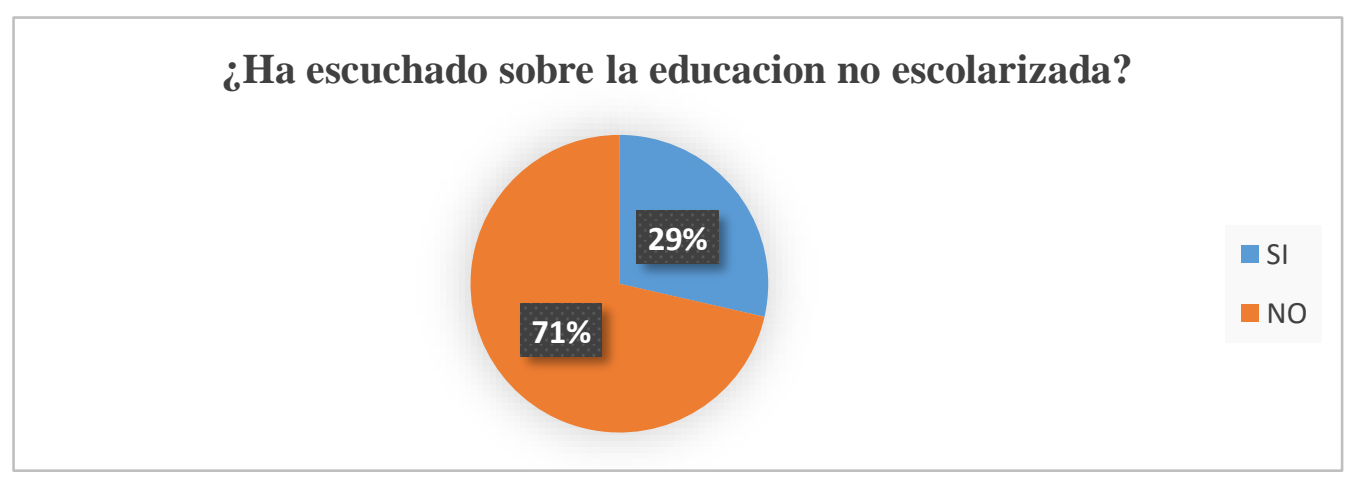

Fuente: Elaboración propia 
Se puede visualizar un porcentaje de $71 \%$ de que las personas no conocen la educación no escolarizada comentan que no ha tenido información sobre el tema, mientras que un $29 \%$ si ha escuchado sobre este nuevo tipo de educación. Palma, Sarmiento y Román (2015) afirma:

se encontró una técnica que utiliza torneos competitivos como un método para la enseñanza y para probar el conocimiento adquirido por los niños en el área de informática elemental, una asignatura que se brinda en el currículo de educación primaria en Eslovaquia (p.634).

Figura 2. Educación en casa

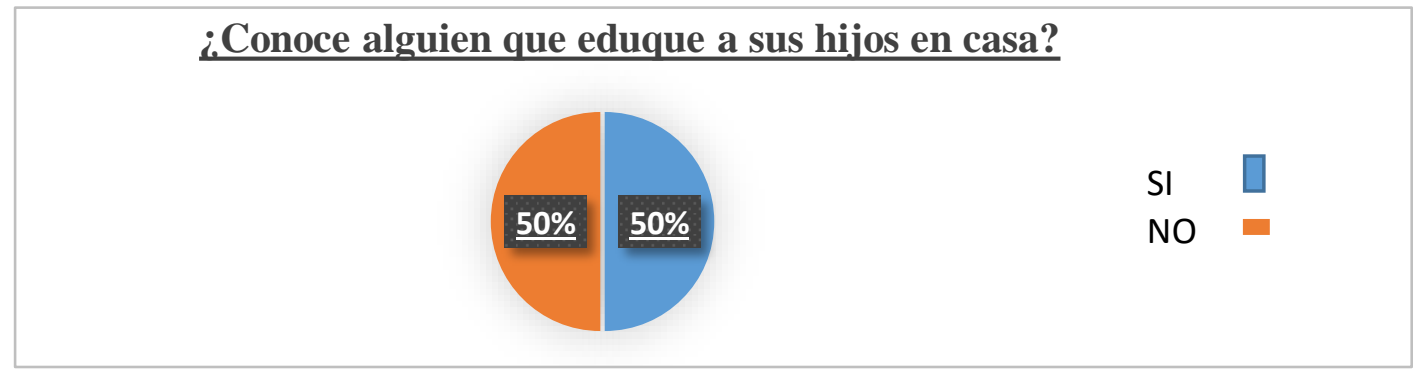

Fuente: Elaboración propia

Respecto a los resultados de las encuestas el $50 \%$ conoce en la población personas que eduque a su hijo en la casa ya que comentan que un lugar seguro, mientras que el 50\% restante no conocen a padres que eduquen a sus hijos en casa ya que consideran que no aprenderán. Casas y Arturo (2014) sostienen:

Los responsables de preservar los derechos de los niños son los padres, tutores y están encargados de proveer lo necesario para propiciar el respeto a la dignidad de la niñez y el ejercicio pleno de sus derechos, así como de otorgar facilidades a los particulares para que coadyuven a su cumplimiento (438).

Figura 3. Legalidad de este tipo de educación

\section{¿Conoce usted si la educacion no escolarizada esta aprobada} por el Ministerio de Educacion?

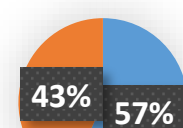

SI

$57 \%$

NO

Fuente: Elaboración propia 
Con respeto si la educación no escolarizada está aprobada por el ministerio, el 57\% de las personas si conocen sobre la educación en casa que debido a esta nueva forma de estudio llegan a obtener se tituló ser bachiller, mientras que el $43 \%$ restante creen que no es legal. Bachiller y Bachiller (2015) afirman:

Con esta experiencia, pretendemos mejorar el proceso de aprendizaje de los alumnos y dotar de mayor realismo la realización de las actividades de la asignatura. Esta actividad se plantea, además, para que los alumnos se familiaricen con la prensa económica y extraigan conclusiones de las noticias que se les presentan asociadas a los temas explicados en clase (p.189).

Figura 4. Educar a sus hijos en casa

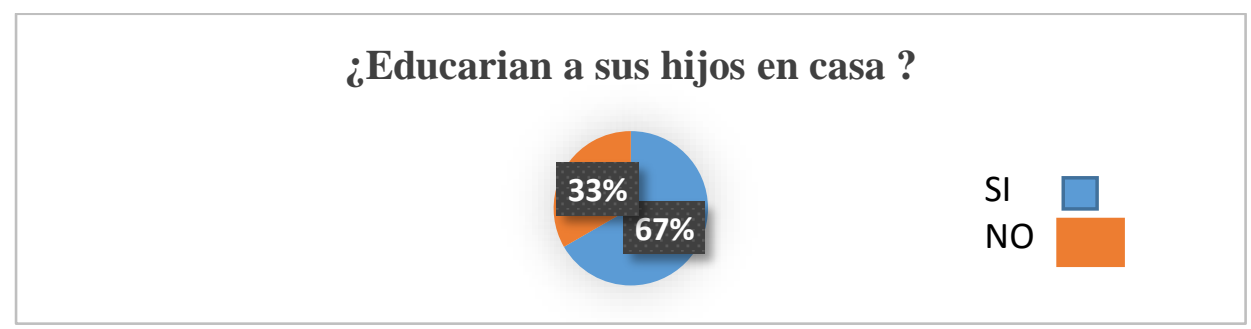

Fuente: Elaboración propia

En la gráfica se puede observar que el $67 \%$ de la población si educaría a su hijo en la casa ya que comentan que nada cambiaria que la forma de estudio es igual en casa como un establecimiento educativo, mientras que el $33 \%$ no educaría a sus hijos en casa por la falta de conocimiento de los beneficios que este tipo de educación posee. Casas y Arturo (2014) afirma "En el cumplimiento de múltiples derechos se está haciendo un análisis de lo anterior se describen algunas acciones que desarrollan en su ejercicio y que propician el cumplimiento de los derechos de los niños entre ellos la educación” (p.189).

Figura 5. Resultados de educar en casa

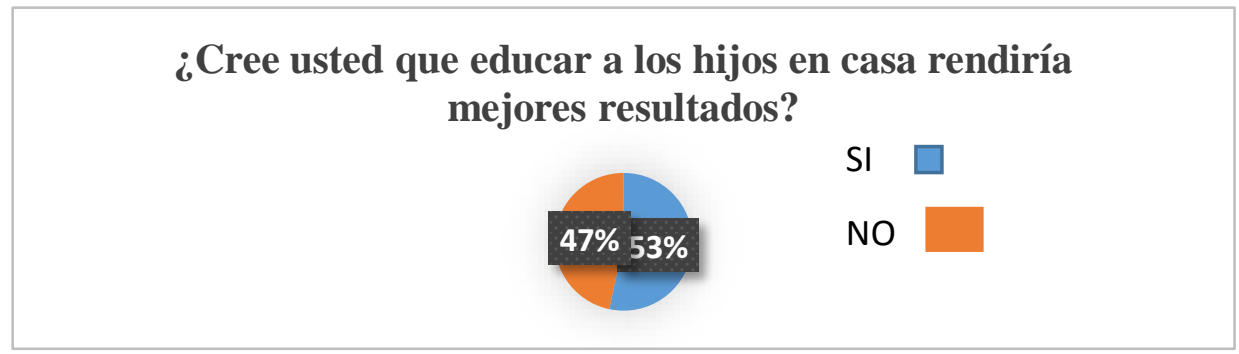

Fuente: Elaboración propia 
Se puede observar en los resultados que los hijos si rendirían mejor estudiando en casa, el 53\% de la población toma como una mejor forma de estudiar para sus hijos ya que tendrán la presencia de sus padres mientras que el $47 \%$ restante no creen que sea mejor para sus hijos. Una nueva oportunidad de estudio de un nivel alto de aprender, conocer, saber y tener la capacidad de mejorar su propósito en la vida. Granados (2015) afirma:

Los estudiantes que se tienen hoy en los salones de clases, no son como los de hace algunos años, ya ellos están inmersos en el manejo de algunas herramientas tecnológicas que le pueden facilitar su proceso de enseñanza-aprendizaje, tienen celulares, calculadoras grabadoras, mini portátiles, tabletas (p.145).

El plan de comunicación consiste en establecer de forma clara los objetivos que se quiere alcanzar en un corto plazo y las estrategias para llegar a cumplir estos objetivos, en esta investigación es de suma importancia, puesto que con este plan se quiere dar a conocer a la población de la Parroquia Octavio Cordero Palacios la importancia de esta nueva modalidad de estudio para el avance de los niños en el campo del estudio.

Tabla 1. Plan de comunicación e información

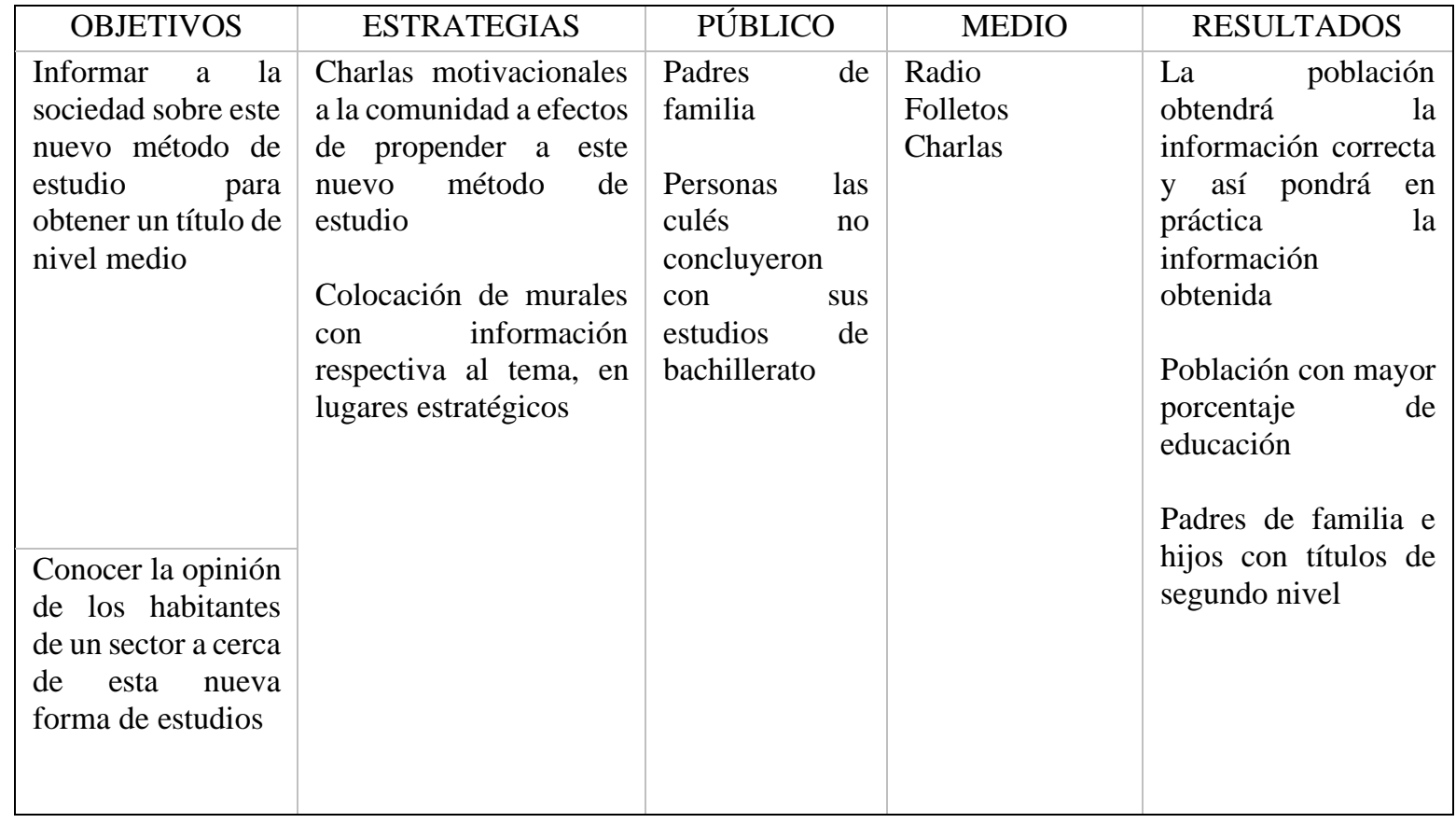

Fuente: Elaboración propia

Este plan de comunicación pretende comunicar e informar al público de la Parroquia Octavio Cordero Palacios, sobre esta modalidad de estudio y tratar de que los padres de familia, hijos y sociedad en general, se interesen por culminar sus estudios bajo este nuevo método, con ello pretender tener una sociedad más equilibrada en cuanto a conocimiento académico se refiere. 


\section{Conclusiones}

El diseñar un plan de comunicación para la Parroquia Octavio Cordero Palacios tiene el propósito de informar a los habitantes sobre esta nueva modalidad de estudio e incentivar a practicarla. La propuesta planteada logro cumplir con el objetivo planteado ciudadanía obtiene una información detallada sobre la educación no escolarizada, se pretende que la mayor parte de las personas perciban esta información de manera adecuada, con el propósito de mejorar los conocimientos, con ello tratar de cumplir con sus objetivos de llegar a obtener el título de nivel medio.

Bajo esta modalidad de estudios, lo importante es que niños y adultos reciban educación, acorde a sus actividades diarias, contando con el apoyo de sus seres queridos, toda sociedad tiende a tener cierto rechazo por lo nuevo, con este estudio se aspira sembrar y motivar a la sociedad a un nuevo cambio, contribuyendo a poner en práctica esta nueva forma de estudio. El aporte del presente estudio, está dirigido hacia los padres de familia y personas que están interesadas en obtener un título de nivel medio en la modalidad de educación no escolarizada, el mismo pretende que la población conozca con mayor exactitud los beneficios que brinda este medio de estudio, el cual no ha sido considerado con la suficiente relevancia por parte de los pobladores de la Parroquia antes mencionada, buscando generar un impacto positivo tanto a corto y largo plazo.

Es importante mencionar que los padres que decidan educar a sus hijos en casa lo hagan de manera responsable, una sociedad bien escolarizada conlleva al desarrollo social y académico de una sociedad.

\section{Referencias Bibliográficas}

Bachiller, P. y Bachiller, A. (2015). Una experiencia docente en los estudios de administración y dirección de empresas: análisis empírico con estudiantes. INNOVAR, 25(55). 185-194. Recuperado de: https://www.redalyc.org/articulo.oa?id=81832838014

Bravo, C. (2009). Como estimular la creatividad en educación. International Journal of Developmental and Educational Psychology, 2(1), 89-97. Recuperado de: https://www.redalyc.org/articulo.oa?id=349832321009

Casas, M. y Abdalá, A. (2014) ¿Por qué debemos conocer los derechos de niñas, niños y adolescentes?35(6).437-439. https://www.redalyc.org/articulo.oa?id=423640350001 
Deobold, B., Meyer, V. D., \& Meyer, W. J. (2006, 12 septiembre). LA INVESTIGACIÓN DESCRÍPTIVA.NOEMAGICOS.https://noemagico.blogia.com/2006/091301-lainvestigaci-n-descriptiva.php

Farrel, J. Manion, C. y Rincón S. (2017). Reinventar la escuela: alternativas radicales exitosas del sur global. Revista Latinoamericana de Estudios Educativos, 47(2), 9-4. Recuperado: https://www.redalyc.org/articulo.oa?id=27052400002

Granados, O. (2015). Las TIC en la enseñanza de los métodos numéricos. Sophia, 11(2). 143154.Recuperado: https://www.redalyc.org/articulo.oa?id=413740778003

Gutiérrez, F. (2014). Inclusión en Educación Superior: Retos y Mitos. Revista Estomatológica Herediana, 24 (1), 3-4. https://www.redalyc.org/articulo.oa?id=421539380001

Ley Orgánica de Educación Intercultural del Ecuador. (2011) LOEI. Recuperado de: http://www.contusguaguas.com/uploads/2/1/0/7/21075456/ley_orga\%CC\%81nica_de _educacio\%CC\%81n_intercultural_y_reglamento.pdf

Ministerio, E.E, (2019). Educación no escolarizada. https://educacion.gob.ec/educacion-noescolarizada/

PressMolero, A. J. (2005, octubre). PLAN DE COMUNICACIÓN concepto, diseño e implementación. Recuperado 21 diciembre, 2019, de http://conocimiento.incae.edu/ES/no_publico/ilgo05/presentaciones/A.MoleroPlanCo municacion.pdf

Maya, E. (2014). Métodos y Técnicas de Investigación (primera edición electrónica). México https://arquitectura.unam.mx/uploads/8/1/1/0/8110907/metodos_y_tecnicas.pdf

Palma, S. Sarmiento, P. y Romane, E. (2015) estado del arte sobre experiencias de enseñanza de programación a niños y jóvenes para el mejoramiento de las competencias matemáticas en primaria 20(65). 607641.Recuperado:https://www.redalyc.org/articulo.oa?id=14035408013

Rodríguez, C. (2019). Homeschooling, la escuela en casa. Web Consultas, 1-3. Recuperado de: https://www.webconsultas.com/bebes-y-niños/educación-infantil/homeschooling$\underline{4609}$

Sampieri, H. Fernandez, C. y Baptiste, P. (2014). Metodología de la Investigación. México D.F, México. Mc Graw Hill Educación.

Sierra, S. (2010) Internacional de Educación. Niñez y Juventud.8(2), 1222-1223.Recuperado: https://www.redalyc.org/articulo.oa?id=77315155032 
Tanaro, M. (2006) Acerca de la afirmación de la igualdad de oportunidades sostenida por la Ley Federal de Educación. Ciencias Sociales.30(10), 139-154.

https://www.redalyc.org/articulo.oa?id=185030099

Tu Gimnasia Cerebral. (2014). Las Encuestas - Qué son, Características, Cómo Hacerlas. Recuperado 22 diciembre, 2019, de http://tugimnasiacerebral.com/herramientas-deestudio/que-es-una- encuesta-características-y-como-hacerlas

Martín, F. (2011), El plan estratégico de comunicación como nuevo modelo de investigación científica universitaria, Dialnet, 101-114

Gutiérrez, J. A. T., Acebrón, L. B., \& Casielles, R. V. (2005). Investigación de mercados: métodos de recogida y análisis de la información para la toma de decisiones en marketing (Ed. rev.). Madrid, España: Parafino.

Valle, J. (2012). Enseñar en casa o en la escuela. La doctrina legal sobre el homeschooling en España. Perfiles Educativos, 34(138), 167-182. https://www.redalyc.org/articulo.oa?id=13224551011

Fonseca, M. (2005). Comunicación Oral: Fundamentos y Práctica Estratégica. México: Pearson educación

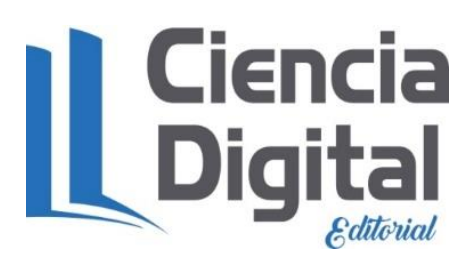




\section{Para citar el artículo indexado}

Luna Altamirano, K. A., Aguilar Aguilar, N., Moreno Guanaquiza, J. V., \& Santos Medina, D. A. (2020). La educación no escolarizada como nueva opción para el bachillerato. Explorador Digital, 4(1), 17-28. https://doi.org/10.33262/exploradordigital.v4i1.1068

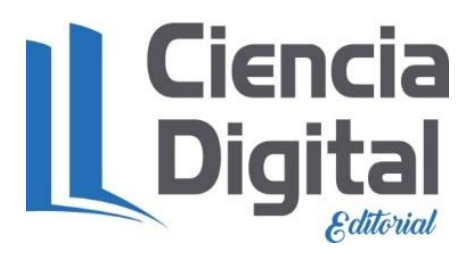

El artículo que se publica es de exclusiva responsabilidad de los autores y no necesariamente reflejan el pensamiento de la Revista Explorador Digital.

El articulo queda en propiedad de la revista y, por tanto, su publicación parcial y/o total en otro medio tiene que ser autorizado por el director o editor de la Revista Explorador Digital.

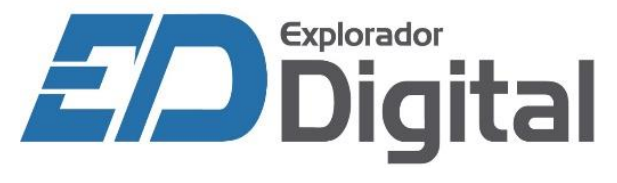

